\title{
Treatments for Acute Bipolar Depression: Meta- analyses of Placebo-controlled, Monotherapy Trials of Anticonvulsants, Lithium and Antipsychotics
}

Authors

Affiliations

\author{
V. Selle ${ }^{1,2}$, S. Schalkwijk ${ }^{1,3}$, G. H. Vázquez ${ }^{1,4}$, R. J. Baldessarini ${ }^{1,5}$
}

International Consortium for Bipolar Disorder Research, McLean Hospital, Belmont, Massachusetts, USA 2Department of Psychiatry, Viarnetto Clinic, Lugano, Switzerland Department of Pharmaceutics, Utrecht Institute for Pharmaceutical Sciences (UIPS), Utrecht University, Utrecht, Netherlands Department of Neuroscience, Palermo University, Buenos Aires, Argentina Department of Psychiatry, Harvard Medical School, Boston, Massachusetts, USA

Key words

anticonvulsants

- antipsychotics

bipolar depression

controlled-trials

lithium

meta-analysis $\begin{array}{ll}\text { received } & 05.09 .2013 \\ \text { revised } & 24.11 .2013 \\ \text { accepted } & 25.11 .2013\end{array}$

Bibliography

DOI http://dx.doi.org/

10.1055/s-0033-1363258

Published online ahead of print:

18 February 2014

Pharmacopsychiatry 2014;

47: 43-52

(c) Georg Thieme Verlag KG

Stuttgart · New York

ISSN 0176-3679

\section{Correspondence}

\section{Dr. R. J. Baldessarini}

Mailman Research Center

McLean Hospital

115 Mill Street

Belmont, MA 02478-9106

USA

Tel.: + 1/617/8553203

Fax: + 1/617/855 3479

rbaldessarini@mclean.harvard.

edu

\section{Abstract}

$\nabla$

Background: Optimal treatments for bipolar depression, and the relative value of specific drugs for that purpose, remain uncertain, including agents other than antidepressants.

Methods: We searched for reports of placebocontrolled, monotherapy trials of mood-stabilizing anticonvulsants, second-generation antipsychotics, or lithium for acute major depressive episodes in patients diagnosed with type I or II bipolar disorder and applied random-effects meta-analysis to evaluate their efficacy, comparing outcomes based on standardized mean drug-placebo differences (SMD) in improvement, relative response rates (RR), and number-needed-to-treat (NNT).

Results: We identified 24 trials of 10 treatments (lasting 7.5 weeks, with $\geq 50$ collaborating sites/trial) that met eligibility criteria: lamotrigine (5 trials), quetiapine (5), valproate (4), 2 each for aripiprazole, olanzapine, ziprasidone, and 1

\section{Introduction}

\section{$\nabla$}

Effective and safe treatment of depressive, dysthymic, and dysphoric or mixed components of bipolar disorders remains among the most challenging problems in modern clinical psychopharmacology [1,2]. Overall, bipolar disorder patients in mid-course or from onset, treated by current community clinical standards, spend approximately half of the weeks of follow-up in symptomatic morbid states, and fully three-quarters of that morbidity is depressive [3]. Depressive components of bipolar disorder contribute importantly not only to long-term morbidity, but also to co-morbidity, disability, and excess mortality $[1,2]$. Despite the pressing need for improved treatments for depressive episodes and the frequent failure of ongoing preventive treatments, remarkably little research has been directed to the problem [2]. Expert treatment recommenda- each for carbamazepine, lithium, lurasidone, and olanzapine-fluoxetine. Overall, pooled drugover-placebo responder-rate superiority (RR) was moderate (29\% [CI: 19-40\%]), and NNT was 8.2 (CI: 6.4-11). By SMD, apparent efficacy ranked: olanzapine + fluoxetine $\geq$ valproate $>$ quetiapine > lurasidone > olanzapine, aripiprazole, and carbamazepine; ziprasidone was ineffective, and lithium remains inadequately studied. Notably, drugs were superior to placebo in only $11 / 24$ trials (5/5 with quetiapine, $2 / 4$ with valproate), and only lamotrigine, quetiapine and valproate had $>2$ trials. Treatment-associated mania-like reactions were uncommon (drugs: 3.7\%; placebo: $4.7 \%$ ).

Discussion: Controlled trials of non-antidepressant treatments for bipolar depression remain scarce, but findings with olanzapinefluoxetine, lurasidone, quetiapine, and perhaps carbamazepine and valproate were encouraging; lithium requires adequate testing.

tions continue to be tentative and inconsistent concerning depressive components of bipolar disorders, but typically ascribe high value to agents usually considered to be mood-stabilizers as well as to some modern antipsychotics [4-7]. Some of the available therapeutic research on these non-antidepressant treatments has been reviewed recently [8-11]. Nevertheless, important uncertainties remain about the relative efficacy and safety of antidepressants, anticonvulsants, lithium salts, second-generation antipsychotics, and several experimental treatments for bipolar depression [2,8-14]. This uncertainty reflects the striking paucity of well and unambiguously designed, controlled trials specifically for bipolar depression.

Given these uncertainties, we collected and analyzed available data concerning the relative efficacy of various anticonvulsants with putative mood-stabilizing properties and second-genera- 
tion antipsychotics, as well as lithium salts, specifically for treatment of acute bipolar depression. We hypothesized that these treatments would vary in the amount of information available as well as in apparent efficacy based on data pooled across trials by meta-analytical methods using different outcome measures.

\section{Methods}

$\nabla$

We performed a comprehensive literature search for reports on treatments for bipolar depression, focusing on randomized, controlled trials (RCTs) of mood-stabilizing anticonvulsants, second-generation antipsychotics, or lithium salts in acute major depressive episodes in patients diagnosed with type I or II BD. We carried out a systematic search [15] of several literature databases (PubMed, PsychInfo, EMBASE, and ClinicalTrials.gov). Search terms included various combinations of "anticonvulsants" (and names of individual agents; carbamazepine, lamotrigine, levetiracetam, oxcarbazepine, valproate), "antipsychotics" (second-generation or atypical, and names of individual antipsychotics: amisulpride, aripiprazole, asenapine, iloperidone, lurasidone, olanzapine, quetiapine, risperidone, ziprasidone); and "lithium” as well as "bipolar", "controlled", "depression”, “efficacy", "randomized", "treatment", and "trial". In addition, we hand-searched citations in identified reports and systematic reviews on this topic. Trial inclusion criteria were: [a] acute phase of major depressive episodes in type I or II BD diagnosed by standard, internationally accepted diagnostic criteria, [b] $\geq 18$ patients/trial; [c] randomized treatment; [d] mood-stabilizing anticonvulsants, second-generation antipsychotics, or lithium salts as monotherapy; [e] placebo control ( \pm other comparators); [f] double-blinded; [g] nominal trial duration $\geq 4$ weeks.

We excluded reports of studies concerning BD patients in episodes other than acute depression, trials involving add-on treatments, special populations (such as geriatric or pediatric patients, or those with known poor treatment responsiveness), and long-term studies of potential prophylactic effects. Abstracts of initially identified reports were screened for possible relevance, and evaluated for meeting our a priori study criteria requirements by independent review of full texts by 2 investigators (VS and SS). As a secondary consideration, several trials of interest that were excluded from the primary meta-analyses due to methodological shortcomings, were considered separately; they included early, small, brief trials of lithium carbonate with crossover designs involving partial placebo controls, or comparisons of bipolar vs. unipolar major depression.

We extracted data from included full reports, including the sex distribution and average age of subjects, treatments and doses, subjects per trial arm and number of collaborating sites, mean baseline depression ratings in each trial arm, and approximate average weeks of treatment. Clinical ratings involved changes in scores on a standardized depression symptom rating scale (usually Montgomery-Åsberg depression rating scale [MADRS] or Hamilton depression rating scale [HDRS] with 17 or 21 items). Outcomes were either improvement (and percentage change) in depression ratings or rates of achieving "response" (usually $\geq 50 \%$ reduction of initial depression symptom ratings). We also recorded reported rates of prematurely dropping out of trials in each arm as well as reported rates of mood switching from depression into mania-like (hypomania, mania, or mixed manic depressive) states.
Analyses included random-effects meta-analyses for individual trials and with pooling for overall assessments and for specific agents. Outcomes involved pooled drug/placebo response rate ratios (RR), standardized mean differences (SMD, as Hedges' $g$ statistic), or response rate differences (RD) used to estimate number-needed-to-treat (NNT, as 1/RD), all with 95\% confidence intervals $(\mathrm{CI})$. In order to manage variance among outcome measures and its impact on rankings of apparent efficacy, we averaged 3 rankings based on RR, SMD, and NNT for each trial, and noted ranking for all 10 agents included for analysis. Correlations were tested with bivariate linear regression $(r)$ or non-parametric Spearman rank correlation $\left(r_{s}\right)$ methods. Potential covariates with SMD were assessed for at least suggestive associations $(p \leq 0.10)$ in preliminary bivariate meta-regression analyses in preparation for multivariate meta-regression analysis. Averages are reported as mean \pm standard deviation (SD), sometimes weighted by subject number. Changes in depression ratings were standardized by subject counts, and variance is reported as SD calculated or imputed from pooled SD from all trials [16]. Statistical analyses used commercial software [Statview. $3^{\circledR}$ (SAS Institute, Cary, NC), and Stata. $10^{\circledR}$ (StataCorp, College Station, TX)].

\section{Results \\ $\nabla$}

\section{Trials identified}

We identified a total of 4915 potentially relevant report titles at initial screening. Based on review of abstracts, 145 reports met eligibility criteria and were considered likely candidates for inclusion. Subsequent exclusions $(121 / 145 ; 83.4 \%)$ were as follows: [a] 97 (66.9\%) trials concerned BD patients in episodes other than acute depression, [b] 13 (8.97\%) were long-term studies of potential prophylactic effects; [c] 7 (4.83\%) involved add-on treatments, [d] 4 (2.76\%) involved special populations. An additional 19 trials did not meet inclusion criteria owing to design limitations but included findings of interest and were considered for comment but not included in primary meta-analyses. In total, 24 trials met all inclusion/exclusion criteria and were included in the primary meta-analytical analyses ( $\bullet$ Table 1) [17-38]. 2 included studies [18,27] had 3 arms comparing 2 different drugs to placebo, and 2 others [24,29] reported on 2 independent trials of the same agents; each of these 4 separate drug-placebo comparisons was included as a separate trial. 5 trials lacked peer-reviewed publications ( 4 for lamotrigine, 1 for valproate), and their data were extracted from 2 pharmaceutical summary trial reports [32,33] and 5 reviews [34-38].

\section{Trial characteristics}

Overall meta-analysis included a total of 7307 unique subjects (4543 randomized to an active agent and 2764 to placebo, adjusting placebo-treated subjects by 484 used in 2 comparisons $[18,27]) ; 16 / 20$ trials $(80.0 \%)$ providing such information involved outpatients, and $4(20.0 \%)$ involved both hospitalized and ambulatory patients. The numbers of collaborating sites/ trial varied widely, from 1 to 110, and averaged 55. Proportions of bipolar disorder types varied, from all bipolar I in $86.5 \%$ of trials, to all bipolar II participants in 1 trial with lamotrigine ( Table 1). 10 treatments were tested, including: aripiprazole (2 trials, at $5-30 \mathrm{mg} / \mathrm{day}$ ); carbamazepine (1 trial, at an average of $452 \mathrm{mg} /$ day); lamotrigine (5 trials; mean dose $220 \pm 48 \mathrm{mg} /$ day); lithium carbonate (1 trial; at $600-1800 \mathrm{mg} /$ day); lurasi- 


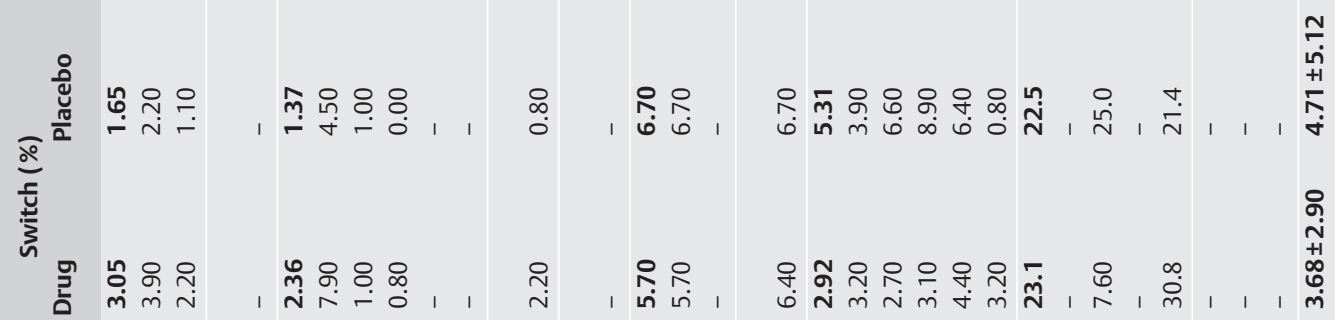

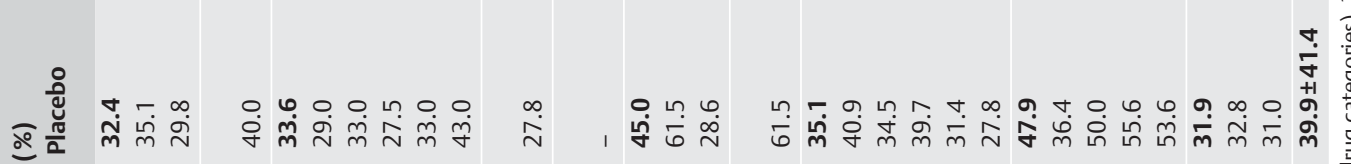
产

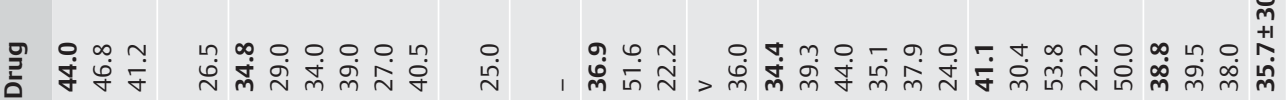

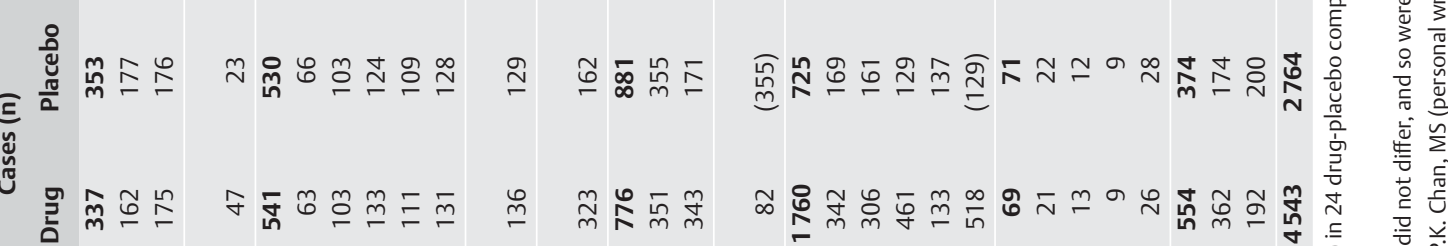

舟芦芦

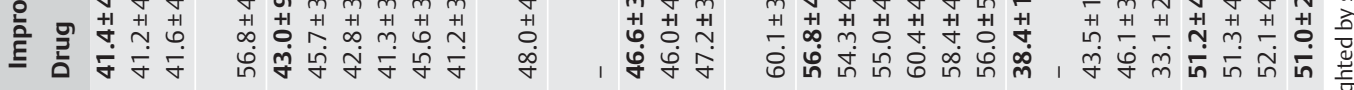

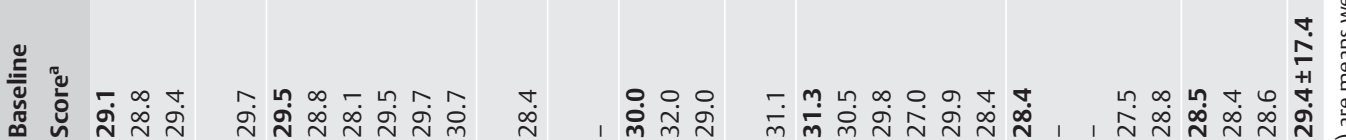

可

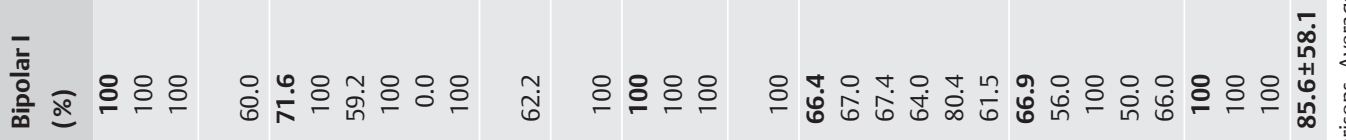

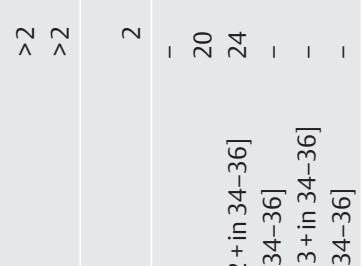


done (1 trial, at $20-60$ or $80-120 \mathrm{mg} /$ day); olanzapine (2 trials, dosed at $5-20 \mathrm{mg} /$ day), olanzapine/fluoxetine combination (1 trial, dosed at $6 / 25,6 / 50$, or $12 / 50 \mathrm{mg} /$ day); quetiapine (5 trials, at 300 or $600 \mathrm{mg} /$ day), valproate, usually as divalproex (4 trials; mean dose $1225 \pm 183 \mathrm{mg} /$ day); and ziprasidone (2 trials dosed at $40-80$ or $120-160 \mathrm{mg} /$ day). Baseline depression ratings based on MADRS or HDRS ranged from 27.0 to 32.0 and were very similar in drug and placebo arms, averaging $29.4 \pm 17.4$ overall. Trial durations ranged from 6 to 10 weeks (weighted mean, $7.5 \pm 4.8$ weeks; $\odot$ Table 1 ).

Weighted average rates of trial non-completion (dropout) were similar in drug (35.7\%) and placebo arms (39.9\%) of the 24 trials, but reasons for dropping out were not provided consistently. With active agents, dropout rates were both highest (53.8\%) and lowest $(22.2 \%)$ in trials with valproate, although only lamotrigine, quetiapine and valproate had more than 2 trials to evaluate (॰ Table 1).

Based on available data, risks of mood switching into hypomania, mania, or mixed states with active agents, as defined in each trial, were highest in one trial with valproate $(30.8 \%)$, moderate with other agents $(0.8-7.6 \%)$, and ranged from $0.0 \%$ to $21.4 \%$ in placebo arms, but were not reported for trials involving carbamazepine, lurasidone, or ziprasidone ( $\bullet$ Table 1 ). Unusually high switch rates appear to be related to particular trials rather than treatments, since switch rates associated with active agents and placebo were highly correlated within trials $(r=0.772$, $p=0.0005)$. Overall, switch rates were slightly, but not significantly, lower with active drugs (3.68 [CI: 2.07-5.29]) than placebo (4.71\% [1.89-7.55]; ○ Table 1 ).

\section{Improvements in depression symptom ratings}

Differences in reported percent improvement in depression symptom ratings were moderate $(51.0 \pm 23.0 \%$ with drugs vs. $39.6 \pm 25.7 \%$ with placebo), and were superior with drug over placebo in all but 2 of 22 comparisons (involving lamotrigine or valproate; 0 Table 1). The overall mean paired difference was small (9.43\% [CI: 5.90-13.0], paired $t=5.55, p<0.001)$, and the median was even smaller (7.30\%; [IQR: 4.00-15.8\%]). Average improvements with antipsychotic drugs were slightly but not significantly greater than with anticonvulsants or lithium (11.5\% [7.03-16.0] vs. $6.91 \%[0.72-13.1] ; t=1.38, p=0.18)$. Average rates of improvement with drugs-minus-placebos ranked: olanzapine-fluoxetine $(22.1 \%$ [ 1 trial] $)>$ quetiapine $(16.9 \%$ [range: 14.1-21.0\%]) > carbamazepine (11.8\% [1 trial]) > olanzapine alone $(7.25 \%$ [6.50-8.00\%]) > valproate $(7.07 \%$ [ -9.90 to $16.5 \%])$ $\geq$ lamotrigine $(6.58 \%[-0.80$ to $18.2 \%])>$ aripiprazole $(3.25$ $[2.50-4.00 \%]) \geq \operatorname{lithium}(3.20 \%[1$ trial $])$.

\section{Meta-analyses}

Based on random-effects meta-analyses, there was a highly significant superiority of active agents over placebo, averaging $29 \%$ [CI: 19-40\%], based on pooled RR value of 1.29 [CI: 1.19-1.40] $(z=6.25, p<0.0001$; 0 Table 2). The pooled RR value for the 4 most effective agents (olanzapine+fluoxetine, lurasidone, quetiapine, and valproate) was 1.47 [CI: 1.32-1.64]; $(z=7.09$ $p<0.0001$; not shown). However, there was considerable heterogeneity of outcomes across trials, such that more than half (54.2\% [13/24]) did not significantly differentiate a test agent from placebo. Rates of failed trials, in descending rank-order based on pooled SMD values ( $\bullet$ Table 3 ) were: aripiprazole $(2 / 2[100 \%])=$ ziprasidone $(2 / 2[100 \%])=$ lithium $(1 / 1[100 \%])$ $>$ lamotrigine $(4 / 5[80 \%])>$ olanzapine alone $(1 / 2[50 \%])=$ valproate
$(2 / 4[50 \%])>$ carbamazepine $(0 / 1[0 \%])>$ lurasidone $(0.1[0 \%])=$ olanzapine + fluoxetine $(0 / 1[0 \%])=$ quetiapine $(0 / 5[0 \%])$.

Separate meta-analyses also were made for each treatment, comparing relative rates of responding to drug vs. placebo (RR), estimated number-needed-to-treat (NNT), and standardized mean drug-placebo difference in improvement of depression ratings (SMD), each measure with confidence intervals for each treatment. These comparisons ( $\bullet$ Table 3 ) indicate similar findings across treatment types with all 3 outcome measures. However, only 5/10 treatments yielded favorable NNT estimates of $<10$ (ranking: olanzapine-fluoxetine [1.8]<carbamazepine $[3.4]<$ valproate $[4.4] \leq$ lurasidone $[4.6] \leq$ quetiapine [5.9]). The 3 outcomes are highly correlated, as expected (Spearman $r_{s}$ values are: RR vs. NNT; 0.946; SMD vs. RR: 0.897; SMD vs. NNT: 0.878; all $p \leq 0.008$ ), but do not yield identical rankings by apparent efficacy. In order to deal with this variation among outcomes, we constructed rankings of apparent efficacy (drug-placebo differences) of the 10 treatments for each of the 3 outcome measures and then averaged them. These averaged rankings by apparent efficacy were as follow: olanzapine-fluoxetine (mean rank= 1.67) $>$ valproate $\quad(2.00)>$ carbamazepine $\quad(3.00)>$ lurasidone (4.00) $>$ quetiapine (4.33) $>$ olanzapine (6.67) > lamotrigine (7.00) $>$ lithium (7.33) > ziprasidone (9.00) > aripiprazole (10.0).

Of note, 5 of the 10 treatments tested did not show statistical superiority of active drug vs. placebo based on pooled SMD (aripiprazole, carbamazepine, lamotrigine, lithium, and ziprasidone), nor did 3 of these show separation by RR (all but carbamazepine and lamotrigine). In addition, 5/10 treatments (all of the preceding agents as well as olanzapine) yielded relatively unfavorable values of NNT $(\geq 10)$. Lithium, aripiprazole, and ziprasidone were not superior to placebo based on all 3 outcome measures ( $\bullet$ Table 3). Findings concerning efficacy measures for specific treatments are also illustrated in representative forest plots of SMD (a) and RR (b) values and their confidence intervals ( $\bullet$ Fig. 1, panels $\mathbf{a}$ and $\mathbf{b}$ ). It is important to emphasize that both in $\odot$ Table 3 and $\odot$ Fig. 1, the 95\% CIs for most treatments overlap, indicting lack of significant separation. These considerations and the small numbers of trials for most agents, indicate the need for caution in attempting to rank treatments by tested efficacy, based on the available data. Moreover, only lamotrigine, quetiapine, and valproate had more than 2 trials each, and 4 agents had only one (carbamazepine, lithium, lurasidone, and olanzapine + fluoxetine; $\bullet$ Table 3).

\section{Covariates of effect size}

The following factors lacked even suggestive covariance with SMD (all $p>0.10$ ), as tested by bivariate meta-regression: [a] the proportion of women participants, [b] mean subject-age, [c] proportion of bipolar-I disorder diagnoses, [d] trial size (subjectcount) and [e] number of collaborating sites, [f] year of reporting, [g] rating scale employed, [h] trial-duration, [i] assessments/ month, [j] dropout rates, and [k] baseline severity score. In addition, [l] the rate of mood switching was suggestively, but again not significantly, associated with SMD (slope $=0.022$ [CI: -0.002 to +0.074$], t=1.98, p=0.07$ ). Accordingly, none of these factors was tested further in multivariate meta-regression analysis. Of note, however, these comparisons are limited by the small number of trials, lack of multiple trials for several treatments, and limited variance of some measures. Given the small numbers of trials involving highly dissimilar agents, we also did not attempt to test for possible publication bias (as with Egger's test or funnel plots). 


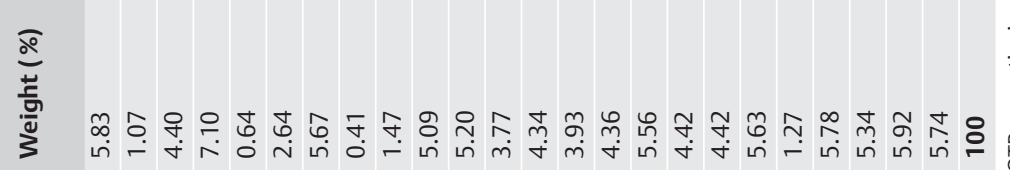

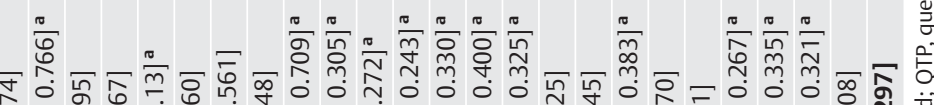

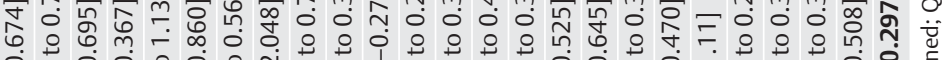

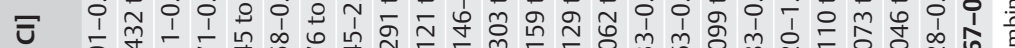

๙ oำ

ڤू

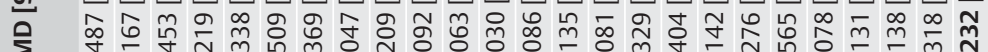

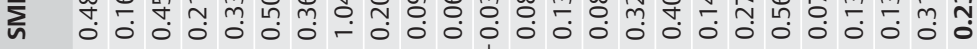

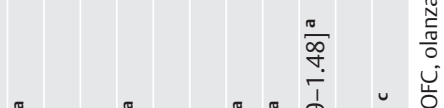

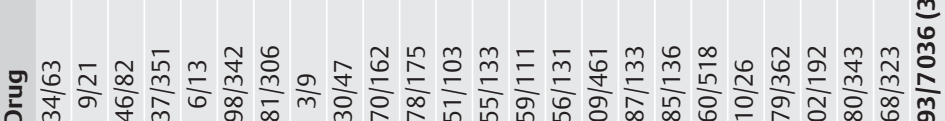

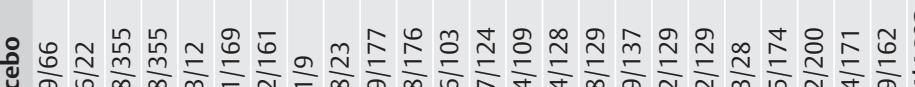

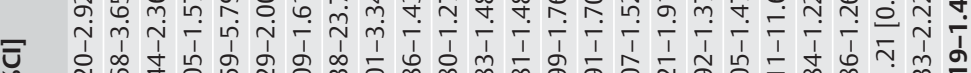

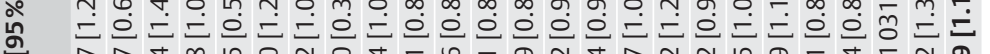

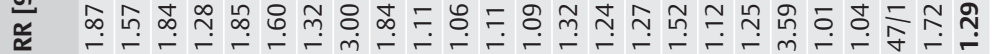

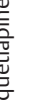
(1) 


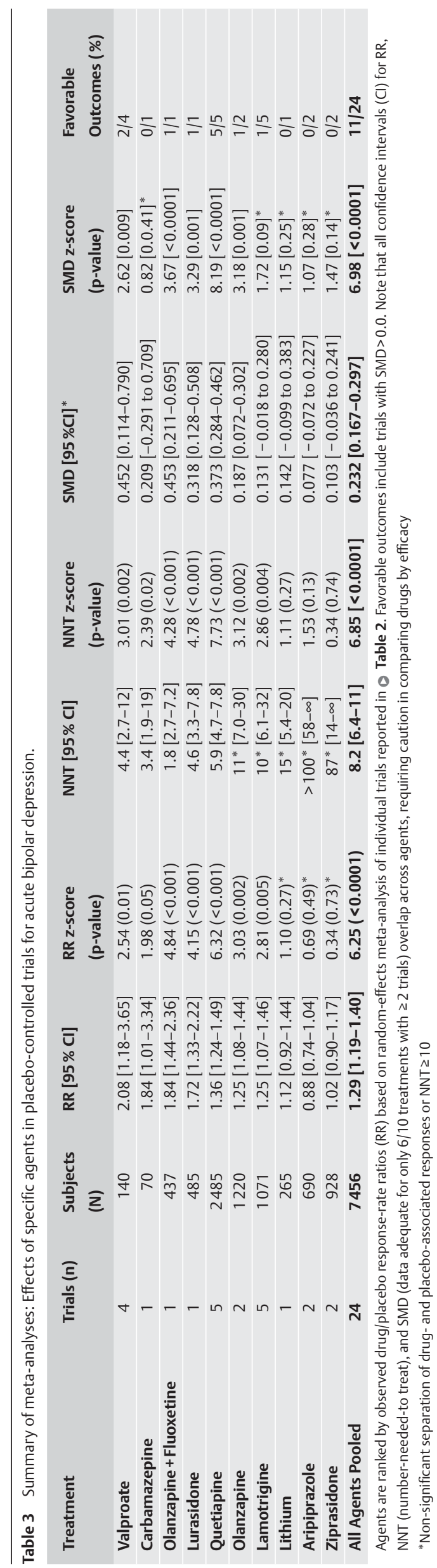

Additional trials considered secondarily for comment

A total of 19 other trials in acute bipolar depression had relevant information but did not meet study criteria for inclusion in the primary meta-analyses. One trial of lamotrigine involving 410 bipolar I patients, without a placebo arm, found this anticonvulsant to be non-significantly less effective than olanzapine + fluoxetine (response rates: 60 vs. $68 \%$; $p=0.07$ ) [39]. Another small trial with bipolar I and II disorder patients, also lacking placebo controls, found similarly small improvements $(21 \% ; p=0.78)$ with lamotrigine or citalopram added to mood stabilizers [40]. In a third trial in 124 bipolar disorder subjects (68\% type I), after 8 weeks, lamotrigine added to lithium treatment was somewhat more effective than placebo ( $\mathrm{RR}=1.63$ [CI: $1.05-2.53$ ]; $z=2.17$, $p=0.03$ ) [41]. These findings are inconclusive regarding the possible efficacy of lamotrigine in acute bipolar depression.

In earlier reviews, 6/9 uncontrolled trials of lithium suggested some clinical benefit in bipolar depressed subjects, as did 8/9 placebo-controlled crossover trials [42-44], but found lithium to be inferior to a tricyclic antidepressant in 3/4 trials [45]. Several of these trials call for further consideration, even though they did not meet inclusion criteria for the primary meta-analyses of this study. We identified 5 relatively small (approximately 16/ trial arm) and brief (10-28 days) trials that specifically considered hospitalized patients with BD depression and compared lithium treatment with placebo in various crossover designs, or compared patients identified with BD vs. unipolar depression [46-50]. Random-effects, meta-analytical summaries of the findings indicated significant superiority of lithium over placebo $(\mathrm{n}=126$ observations; $\mathrm{RR}=4.85 ; \mathrm{CI}: 1.54-15.3 ; z=2.70, p=0.007$ $[46,47,49,50])$ and in BD vs. unipolar depression $(n=155$; $\mathrm{RR}=2.40$; CI: $1.66-3.48 ; z=4.64, p=0.005[46,48-50])$. These few trials underscore the paucity of research on effects of lithium in acute bipolar depression using adequate trial designs.

With carbamazepine, a small $(\mathrm{N}=7)$, early crossover trial was inconclusive [51]. A second crossover trial involving 24 bipolar I or II disorder patents vs. 11 unipolar major depression cases found significantly greater improvement in the bipolar cases (62 vs. $45 \%$ ) [52].

One trial without a placebo control found no difference between topiramate and bupropion in 38 depressed BD patients [53]. In 32 bipolar depressed patients (72\% type I) adding levetiracetam to various mood stabilizers yielded non-significantly $(12 \%)$ worse outcomes than with placebo [54].

An open-label, uncontrolled trial of aripiprazole in 31 bipolar disorder patients was inconclusive as well as being associated with a substantial dropout rate (29\%), mainly owing to adverse effects [55]. Another uncontrolled, 84-day study of 30 bipolar disorder patients given aripiprazole (up to $40 \mathrm{mg} /$ day) to augment other treatments also was inconclusive [56].

Finally, adding risperidone, paroxetine, or both to ongoing mood stabilizer treatment without placebo controls yielded similar, small benefits in small numbers of patients with treatmentresistant bipolar depression [57].

\section{Discussion}

Findings in this review of 24 randomized, placebo-controlled comparisons of non-antidepressant treatments for acute bipolar depression are consistent with other recent reviews of portions of this research topic in indicating both limited research and modest efficacy of most treatments tested [8-11]. Remarkably 

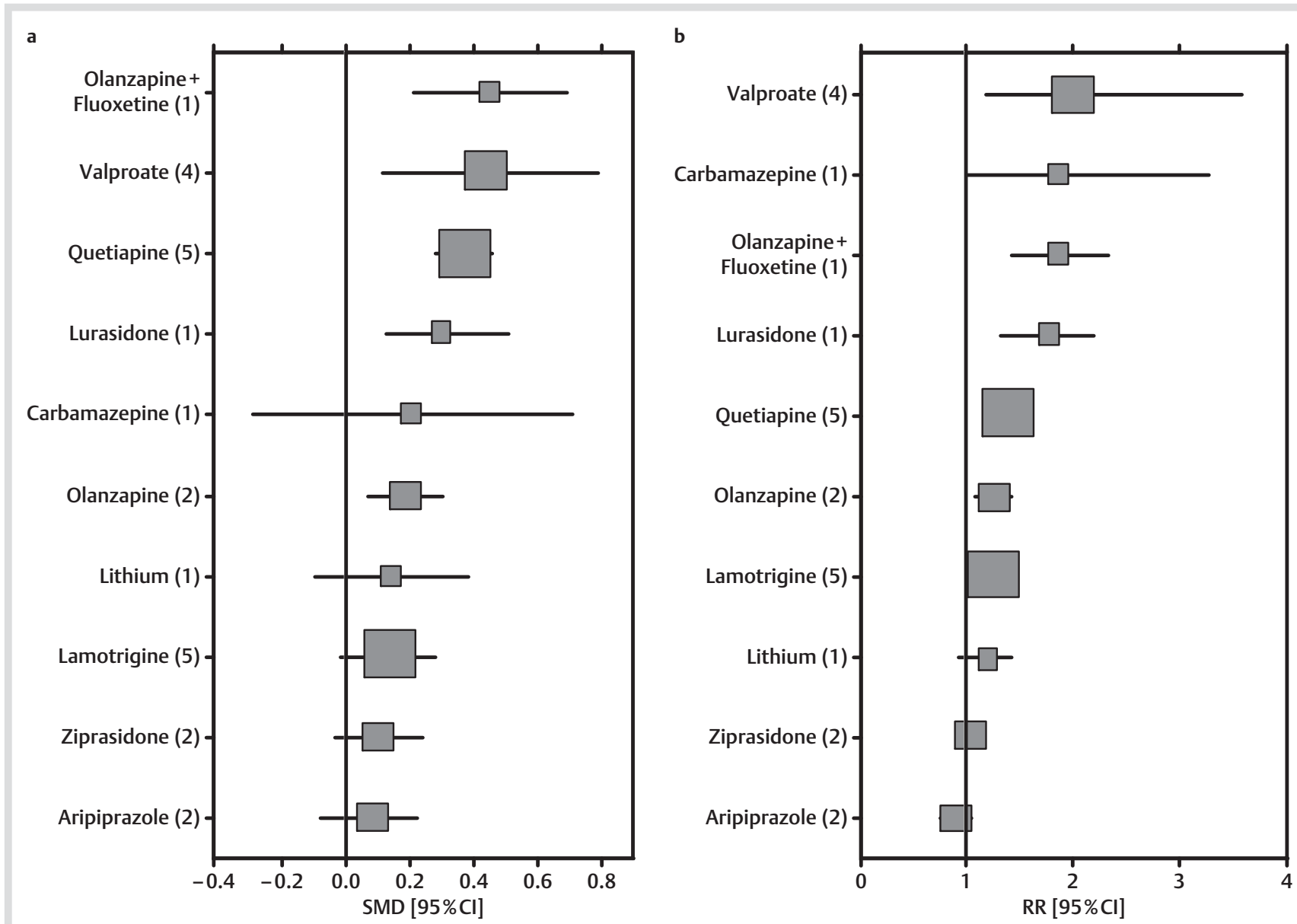

Fig. 1 Forest plot of results of random-effects meta-analysis of findings concerning drug vs. placebo comparisons (a standardized mean difference [SMD], b responder-rate ratio $[R R]$, both with $95 \%$ confidence intervals $[\mathrm{CI}]$ ), pooled for individual agents, based on 24 randomized, placebo-controlled trials of mood-stabilizing anticonvulsants, second-generation antipsychotic agents, or lithium carbonate in acute bipolar major depression ( $(\bullet$ Table 3). Drugs and their trial-counts (2-5) in parentheses are on the $y$-axis. The symbols are sized in proportion to weight (based on trial counts) for each agent; horizontal bars are computed $\mathrm{Cls}$; vertical solid lines are null values $(S M D=0.0 ; R R=1.0)$. Effects of individual treatments are not clearly differentiated owing to overlapping $\mathrm{Cls}$, but aripiprazole, lamotrigine, lithium, and ziprasidone were not significantly superior to placebo by one or the other outcome measure.

few such trials could be identified, and very few treatments (only lamotrigine, quetiapine, and valproate) have been tested in more than one or 2 trials. We also found few additional, relevant trials, including of lithium, that could not be included in primary meta-analyses owing to methodological shortcomings [39-57]. Effects of antidepressants in bipolar depression also have been reviewed extensively $[14,58]$.

A noteworthy observation arising from this review is that rankings of specific treatments by apparent efficacy varied with the outcome measure employed (raw \% improvement, RR, SMD, or NNT; $\odot$ Table 3), but differences tended to be minor ( $\bullet$ Fig. 1). These outcome measures compare ratings of clinical changes with drugs vs. placebo somewhat differently: RR and NNT pertain to the proportion of persons attaining a criterion level of response, whereas SMD and raw percent improvement pertain to changes in symptomatic ratings of illness severity.

Overall, the evidence reviewed here indicates statistical superiority of active agents over placebo controls in only half of reported trials ( $\odot$ Table 2, 3). Outcomes with aripiprazole, lamotrigine, and lithium failed to support efficacy by any reported outcome measure, all but RR for lamotrigine, and results from a single trial of carbamazepine were equivocal by RR and non- significant by SMD ( $\odot$ Table 3). Outcomes among other, apparently effective, non-antidepressants varied widely, from relatively high pooled measures of efficacy with lurasidone, olanzapine + fluoxetine, quetiapine and valproate $(S M D=0.318$ 0.452 ; $R R=1.36-2.08$; NNT $<6$ ) to more moderate values with olanzapine alone $(S M D=0.187 ; R R=1.25 ; N N T=11 ; \diamond$ Table 3$)$. However, even treatments with relatively favorable results were: [a] limited to single trials (lurasidone, olanzapine+fluoxetine), [b] not significantly effective by some outcome measures (olanzapine: high NNT; lamotrigine: low SMD), or [c] had a high proportion of trials with negative findings (valproate: 2/4; - Table 2, 3). This body of evidence provides some encouraging leads, but does not establish consistent and unambiguous evidence of high levels of efficacy of potential treatments for acute bipolar depression. A possible exception is the atypical antipsychotic agent quetiapine, which has been studied in 5 placebocontrolled trials, with statistical superiority to placebo in all trials ( $\odot$ Table 2, 3). However, even this promising treatment had modest effect sizes (e.g., drug vs. placebo average response rate difference with large placebo-associated responses, $16.2 \%$ [56.8-40.6\%]; $\odot$ Table 1) and it may be risky for long-term use 
owing to its strong association with weight gain and metabolic syndrome [59].

The evidence reviewed was remarkably inconsistent or unfavorable and poorly studied for several treatments for which better effects might have been expected ( $\bullet$ Table 2,3 ). Inconsistency is noteworthy for valproate and contrasts to its wide empirical application in various phases of bipolar disorder, including depression $[37,38]$. Similarly, lithium, too, is widely employed $[2,60,61]$, despite having virtually no research support of efficacy in acute bipolar depression ( $\bullet$ Table 3 ), despite some encouraging findings in trials that did not meet study inclusion criteria. In addition, the performance of lamotrigine was uneven ( $\bullet$ Table 1, 3), contrasting to its regulatory approval for longterm treatment of bipolar depressive and manic recurrences [2]. Moreover, lamotrigine usually is administered in slowing increasing doses to limit risks of dermatological reactions, making it difficult to employ in acute phases of bipolar disorder [2]. Of other agents considered, carbamazepine and lurasidone, with only one trial each, appear to be promising and require more study, and further study of lithium would be of interest.

By comparison with the present findings regarding effective non-antidepressant treatments (pooled RR for the most favorable treatments, lurasidone, olanzapine + fluoxetine, quetiapine, and valproate: 1.47 [CI: $1.32-1.64]$ ), a recent meta-analysis of 10 placebo-controlled trials of antidepressants in bipolar depression yielded a pooled RR of 1.43 (CI: 1.11-1.48) [10]. This outcome was unexpectedly similar to findings in a comprehensive meta-analysis of 142 placebo-controlled trials of antidepressants in unipolar major depression (pooled RR=1.42 [CI: $1.38-$ 1.48]), the standard indication for antidepressants. This comparison suggests a lack of major difference in response to antidepressants in the 2 types of depressive illnesses [62], or of clear superiority of anticonvulsants and antipsychotics vs. antidepressants in bipolar depression, despite their typical recommendation as options of first-choice for this indication [7]. However, the place of antidepressants in the treatment of bipolar depression remains controversial and unresolved [2,14,57]. If some relatively favorable results reported here for non-antidepressants ( $\bullet$ Table 3) can be replicated consistently, it might be that some anticonvulsants and modern antipsychotics are somewhat superior to antidepressants for the treatment of bipolar depression. They also may be somewhat safer: rates of treatment-emergent mania-like states were uncommon in the trials reviewed and slightly lower with some active treatments than with placebo ( $\bullet$ Table 1 ).

A final question requiring comment is why there are so few controlled trials of treatments for bipolar depression, despite the introduction of lithium carbonate, antipsychotics, and antidepressants into psychiatric therapeutics over a half-century ago. Antidepressants, though extraordinarily widely used to treat depressive phases of bipolar disorder $[2,14,60]$, tend to be avoided in the treatment of type I bipolar disorder patients in particular $[63,64]$. This tendency and the striking paucity of controlled trials in bipolar depression probably reflect concerns about risks associated with excessive mood elevation - a concern no doubt shared by clinicians, patients, and potential pharmaceutical trial sponsors $[63,64]$. Such concerns appeared not to be relevant to treatment with most non-antidepressant agents, including olanzapine combined with fluoxetine, as observed switch-rates were 3.7-4.7\%, albeit for relatively brief exposure times ( $\bullet$ Table 1 ). In addition, an emerging impression is that risks of mood-switching in bipolar disorder patients, including of type I, during antidepressant treatment, probably are much less than is widely assumed, and not much greater than the substantial spontaneous rates [65]. Another important basis for the paucity of treatment trials in bipolar depression may well be the highly questionable assumption that treatment efficacy and safety in unipolar major depression syndrome can support generalization to all forms of depression [2,14].

Limitations of this study are profound, and reflect the very limited numbers of reported, controlled trials of treatments for bipolar depression. If there is publication bias on this topic, it is likely to represent selection of relatively favorable trials, despite the generally modest findings encountered [66].

In conclusion, we found some evidence to support at least moderate efficacy of some anticonvulsant and antipsychotic agents in acute bipolar depression, but with very few trials for most treatments, inconsistent performance for 2 of only 3 agents with multiple trials (lamotrigine and valproate, but not quetiapine), and inadequate testing of carbamazepine and lithium, in particular. This review underscores the remarkable conclusion that evidence regarding the possible value of non-antidepressant treatments for acute bipolar depression remains scarce and largely inconclusive - in contrast to the compelling clinical and public health nature of the problem, and prevalent recommendations of mood-altering anticonvulsants and modern antipsychotics as first-line treatment options. The present observations strongly indicate the pressing need for additional treatment research in this severe, but surprisingly poorly studied disorder. In addition to adequate trials for typical cases of bipolar depression, more research is required to test treatment responses in cases of bipolar depression in types I and II bipolar disorder, types with sub-clinical hypomania ("spectrum"), and those with psychotic or mixed features, as well as to clarify the relative efficacy and safety of specific combinations and doses of treatments, and to establish safe and effective long-term treatments aimed at preventing recurrences of bipolar depression. Our general conclusion is that bipolar depression remains one of the most pressing, inadequately addressed problems in contemporary psychiatric therapeutics.

\section{Acknowledgments}

$\nabla$

Supported, in part, by a grant from the Bruce J. Anderson Foundation and by the McLean Private Donors Research Fund (to RJB).

\section{Conflict of Interest \\ $\nabla$}

No author or immediate family member holds equity positions or other financial arrangements with commercial entities that might appear to represent conflicts of interest with the material presented.

\section{References}

1 Baldessarini RJ, Vieta E, Calabrese JR et al. Bipolar depression: overview and commentary. Harv Rev Psychiatry 2010; 18: 143-157

2 Baldessarini RJ. Chemotherapy in Psychiatry. third edition. New York: Springer Press; 2013

3 Baldessarini RJ, Salvatore P, Khalsa H-MK et al. Morbidity in 303 firstepisode bipolar I disorder patients. Bipolar Disord 2010; 12: 264-270

4 Fountoulakis KN, Kasper S, Andreasen O et al. Efficacy of pharmacotherapy in bipolar disorder: report by the WPA section on pharmacopsychiatry. Eur Arch Psychiatry Clin Neurosci 2012; 262 (Suppl 1): 1-48 
5 Malhi GS, Bargh DM, McIntyre R et al. Balanced efficacy, safety, and tolerability recommendations for the clinical management of bipolar disorder. Bipolar Disord 2012; 14 (Suppl 1): 1-21

6 Samalin L, Llorca PM. Methodological approach to inter "guideline" variability in the management of bipolar disorders (French). Encephale 2012; 38: 126-132

7 Yatham LN, Kennedy SH, Parikh SV et al. Canadian Network for Mood and Anxiety Treatments (CANMAT) and International Society for Bipolar Disorders (ISBD) collaborative update of guidelines for management of patients with bipolar disorder: 2013. Bipolar Disord 2013; 15: $1-44$

8 Cruz N, Sanchez-Moreno JH, Torres $F$ et al. Efficacy of modern antipsychotics in placebo-controlled trials in bipolar depression: metaanalysis. Int J Neuropsychopharmacol 2010; 13: 5-14

9 Vieta E, Locklear J, Günther $O$ et al. Treatment options for bipolar depression: systematic review of randomized, controlled trials. J Clin Psychopharmacol 2010; 30: 579-590

10 Kemp DE, Ganocy SJ, Brecher $M$ et al. Clinical value of early partial symptomatic improvement in the prediction of response and remission during short-term treatment trials in 3369 subjects with bipolar I or II depression. J Affect Disord 2011; 130: 171-179

11 Cerullo MA, Strakowski SM. Systematic review of the evidence for the treatment of acute depression in bipolar I disorder. CNS Sprectr 2013; 18: $199-208$

12 Reinares $M$, Rosa AR, Franco $C$ et al. Systematic review on the role of anticonvulsants in the treatment of acute bipolar depression. Int J Neuropsychopharmacol 2013; 16: 485-496

13 Tondo L, Baldessarini RJ, Vázquez $G$ et al. Clinical responses to antidepressants among 1036 acutely depressed patients with bipolar or unipolar major affective disorders. Acta Psychiatr Scand 2013; 127: 355-364

14 Vázquez GH, Tondo L, Undurraga J et al. Overview of antidepressant treatment in bipolar depression. Intl J Neuropsychopharmacol 2013; 22: $1-13$

15 Moher D, Hopewell S, Schulz KF et al. CONSORT 2010 explanation and elaboration: updated guidelines for reporting parallel group randomized trials. BMJ 2010; 340: c869

16 Furukawa TA, Barbui C, Cipriani A et al. Imputing missing standard deviations in meta-analyses can provide accurate results. J Clin Epidemiol 2006; 59: 7-10

17 Calabrese JR, Bowden VL, Sachs GS et al. Double-blind placebo-controlled study of lamotrigine monotherapy in outpatients with bipolar-1 depression. J Clin Psychiatry 1999; 60: 79-88

18 Tohen M, Vieta E, Calabrese J. Efficacy of olanzapine and olanzapinefluoxetine combination in the treatment of bipolar I depression. Arch Gen Psychiatry 2003; 60: 1079-1088

19 Calabrese JR, Keck PE, Macfadden W. Randomized, double-blind, placebo controlled trial of quetiapine in the treatment of bipolar I or II depression. Am J Psychiatry 2005; 162: 1351-1360

20 Davis $L L$, Bartolucci A, Perry F. Divalproex in the treatment of bipolar depression: a placebo-controlled study. J Affect Disord 2005; 85: 259-266

21 Thase ME, Macfadden W, Weisler RH. Efficacy of quietapine monotherapy in bipolar I and II depression: double-blind, placebo-controlled (BOLDER II) study. J Clin Psychopharmacol 2006; 26: 600-609

22 Ghaemi SN, Gilmer WS, Goldberg JF et al. Divalproex in the treatment of acute bipolar depression: preliminary double-blind, randomized, placebo-controlled pilot study. J Clin Psychiatry 2007; 68: 1840-1844

23 Zhang ZJ, Kang WH, Tan QR et al. Adjunctive herbal medicine with carbamazepine for bipolar disorders: double-blind, randomized, placebo-controlled study. J Psychiatr Res 2007; 41: 360-369

24 Thase ME, Jonas A, Khan A. Aripiprazole monotherapy in non-psychotic bipolar I depression: results of 2 randomized, placebo-controlled studies. J Clin Psychopharmacol 2008; 28: 13-20

25 McElroy SL, Richard HW, Chang W. Double-blind, placebo-controlled study of quetiapine and paroxetine as monotherapy in adults with bipolar depression (EMBOLDEN II). J Clin Psychiatry 2010; 71: 163-174

26 Suppes T, Datto C, Minkwitz M et al. Effectiveness of the extended release formulation of quetiapine monotherapy for the treatment of acute bipolar depression. J Affect Disord 2010; 121: 106-115

27 Young AH, McElroy SL, Bauer M. Double-blind, placebo-controlled study of quetiapine and lithium monotherapy in adults in the acute phase of bipolar depression (EMBOLDEN I). J Clin Psychiatry 2010; 71: $150-162$

28 Muzina DJ, Gao K, Kemp DE et al. Acute efficacy of divalproex-sodium vs. placebo in mood stabilizer-naive bipolar I or II depression: doubleblind, randomized, placebo-controlled trial. J Clin Psychiatry 2011; 72: 813-819
29 Lombardo I, Sachs G, Kolluri S et al. Two 6-week, randomized, doubleblind, placebo-controlled studies of ziprasidone in outpatients with bipolar I depression: did baseline characteristics impact trial outcome? J Clin Psychopharmacol 2012; 32: 470-478

30 Tohen M, McDonnell DP, Case M et al. Randomized, double-blind, placebo-controlled study of olanzapine in patients with bipolar I depression. Br J Psychiatry 2012; 201: 376-382

31 Loebel A, Cucchiaro J, Silva $R$ et al. Lurasidone monotherapy in the treatment of bipolar I Depression: randomized, double-blind, placebocontrolled study. Am J Psychiatry 2013 Epub ahead of print (30 Oct)

32 Glaxo-SmithKline Corporation. Multicenter, double-blind, placebocontrolled, flexible dose (100-400 mg) 10 week evaluation of the safety and efficacy of Lamictal (lamotrigine) in the treatment of a major depressive episode in patients with bipolar disorder (summary report of trial SCAA2010, updated 2005, 5 pp). Accessible at: http://www.gsk-clinicalstudyregister.com/result_detail.jsp?proto colId = SCAA2010\&studyId $=6$ FEAE60A-D3CE-4B66-98810EB17D60632B\&compound = lamotrigine (accessed 1 March 2013)

33 Glaxo-SmithKline Corporation. Multicenter, double-blind, placebocontrolled, fixed dose evaluation of the safety, efficacy and tolerability of Lamictal (lamotrigine) in the treatment of a major depressive episode in patients with type I bipolar disorder (summary report of trial SCA40910, updated 2005, 6 pp). Accessible at: http://www.gsk-clinicalstudyregister.com/result_detail. jsp ? protocolId = SCA40910\&studyId = 08FF5601-AD4E-4D59-B8AA652E9448555A\&compound = lamotrigine (accessed 1 March 2013)

34 Goldsmith DR, Wagstaff AJ, Ibbotson T et al. Lamotrigine: review of its use in bipolar disorder. Drugs 2003; 63: 2029-2050

35 Calabrese JR, Huffman RF, White RL et al. Lamotrigine in the acute treatment of bipolar depression: results of five double-blind, placebocontrolled clinical trials. Bipolar Disord 2008; 10: 323-333

36 Geddes JR, Calabrese JR, Goodwin GM. Lamotrigine for treatment of bipolar depression: independent meta-analysis and meta-regression of individual patient data from five randomized trials. Br J Psychiatry 2009; 194: 4-9

37 Bond DJ, Lam RW, Yatham LN. Divalproex sodium vs. placebo in the treatment of acute bipolar depression: systematic review and metaanalysis. J Affect Disord 2010; 124: 228-234

38 Smith LA, Cornelius VR, Azorin JM et al. Valproate for the treatment of acute bipolar depression: systematic review and meta-analysis. J Affect Disord 2010; 122: 1-9

39 Brown EB, McElroy SL, Keck PE Jr et al. Seven-week, randomized, double-blind trial of olanzapine/fluoxetine combination vs. lamotrigine in the treatment of bipolar-I depression. J Clin Psychiatry 2006; 67: 1025-1033

40 Schaffer A, Zuker P, Levitt A. Randomized, double-blind pilot trial comparing lamotrigine versus citalopram for the treatment of bipolar depression. J Affect Disord 2006; 96: 95-99

41 Van der Loos ML, Mulder PG, Hartong EG et al. Efficacy and safety of lamotrigine as add-on treatment to lithium in bipolar depression: multicenter, double-blind, placebo-controlled trial. J Clin Psychiatry 2009; 70: 223-231

42 Mendels J. Lithium in the treatment of depression. Am J Psychiatry 1976; 133: 373-378

43 Zornberg GL, Pope HG Jr. Treatment of depression in bipolar disorder: new directions for research. J Clin Psychopharmacol 1993; 13: 397-408

44 Bhagwagar Z, Goodwin GM. Role of lithium in the treatment of bipolar depression. Clin Neurosci Res 2002; 2: 222-227

45 Yatham LN, Calabrese JR, Kusumakar V. Bipolar depression: criteria for treatment selection, definition of refractoriness, and treatment options. Bipolar Disord 2003; 5: 85-97

46 Goodwin FK, Murphy DL, Bunney WE. Lithium carbonate treatment in depression and mania. Arch Gen Psychiatry 1969; 21: 486-496

47 Stokes PE, Stoll PM, Shamoian CA et al. Efficacy of lithium as acute treatment of manic-depressive illness. Lancet 1971; 1: 1319-1325

48 Goodwin RK, Murphy DL, Dunner DL et al. Lithium response in unipolar vs. bipolar depression. Am J Psychiatry 1972; 129: 76-79

49 Noyes RJr, Dempsey GM. Lithium treatment of depression. Compr Psychiatry 1974; 15: 187-193

50 Donnelly EF, Goodwin FK, Waldman IN et al. Prediction of antidepressant responses to lithium. Am J Psychiatry 1978; 135: 552-556

51 Ballenger JC, Post RM. Carbamazepine in manic-depressive illness: a new treatment. Am J Psychiatry 1980; 137: 782-790

52 Post RM, Uhde TW, Roy-Byrne PP et al. Antidepressant effects of carbamazepine. Am J Psychiatry 1986; 143: 29-34 
53 McIntyre RS, Mancini DA, McCann S et al. Topiramate vs. bupropion-SR when added to mood stabilizer therapy for the depressive phase of bipolar disorder: preliminary single-blind study. Bipolar Disord 2002; 4: 207-213

54 Saricicek A, Maloney K, Muralidharan A et al. Levetiracetam in management of bipolar depression: randomized, double-blind, placebocontrolled trial. J Clin Psychiatry 2011; 72: 744-750

55 McElroy SL, Suppes T, Frye $M$ et al. Open-label aripiprazole in the treatment of acute bipolar depression: prospective pilot trial. J Affect Disord 2007; 101: 275-281

56 Ketter AT, Wang PW, Chandker RA et al. Adjunctive aripiprazole in treatment resistant bipolar depression. Ann Clin Psychiatry 2006; 18 : 169-172

57 Shelton RC, Stahl SM. Risperidone and paroxetine given singly and in combination for bipolar depression. J Clin Psychiatry 2004; 65: 1715-1719

58 Pacchiarotti I, Bond DJ, Baldessarini RJ et al. International Society for Bipolar Disorders (ISBD) task-force report on antidepressant use in bipolar disorders. Am J Psychiatry 2013 in press

59 Centorrino F, Masters G, Baldessarini RJ et al. Metabolic syndrome in psychiatrically hospitalized patients treated with antipsychotics and other psychotropics. Hum Psychopharmacol 2012; 27: 521-526
60 Baldessarini RJ, Henk HJ, Sklar AR et al. Psychotropic medications for bipolar disorder patients in the United States: polytherapy and adherence. Psychiatr Serv 2008; 59: 1175-1183

61 Vieta E, Langosch JM, Figueira ML et al. Clinical management and burden of bipolar disorder: results from a multinational longitudinal study (WAVE-bd). Int J Neuropsychopharmacol 2013; 13: 1-14

62 Undurraga J, Baldessarini RJ. Randomized, placebo-controlled trials of antidepressants for acute major depression: thirty-year meta-analytic review. Neuropsychopharmacology 2012; 37: 851-864

63 Lorenzo LS, Vázquez GH, Zaratiegui RM et al. Characteristics of bipolar disorder patients given antidepressants. Hum Psychopharmacol 2012; 27: 486-491

64 Undurraga K, Baldessarini RJ, Valenti M et al. Bipolar depression: clinical correlates of receiving antidepressants. J Affect Disord 2012; 139: 89-93

65 Tondo L, Vázquez GH, Baldessarini RJ. Mania associated with antidepressant-treatment: comprehensive meta-analytic review. Acta Psychiatr Scand 2010; 121: 404-414

66 Turner EH, Matthews AM, Linardatos E et al. Selective publication of antidepressant trials and its influence on apparent efficacy. $\mathrm{N}$ Engl J Med 2008; 358: 252-260 